\title{
Evaluation of Interfascial Plane and Pericapsular Nerve Blocks to the Shoulder Joint: A Preliminary Analysis of Shoulder Anterior Capsular Block
}

\author{
Felice Galluccio (D) · Mario Fajardo Perez • Ece Yamak Altinpulluk (D) • \\ Jin-De Hou (D) · Jui-An Lin (D)
}

Received: July 9, 2021 / Accepted: September 7, 2021 / Published online: October 20, 2021

(C) The Author(s) 2021

\begin{abstract}
Introduction: The aim of this study is to verify if the shoulder anterior capsular block (SHAC), combined with other nerve blocks, is effective in relieving shoulder pain, avoiding motor block and allowing an early rehabilitation program.
\end{abstract}

Methods: Seventy-five consecutive patients with painful shoulder were treated with the

Supplementary Information The online version contains supplementary material available at https:// doi.org/10.1007/s40122-021-00326-0.

F. Galluccio · M. Fajardo Perez · E. Yamak Altinpulluk

MoMaRC Morphological Madrid Research Center, Madrid, Spain

F. Galluccio

Division of Rheumatology, Medical-Geriatric

Department, University Hospital AOU Careggi,

Florence, Italy

F. Galluccio · J.-A. Lin

Center for Regional Anesthesia and Pain Medicine,

Wan Fang Hospital, Taipei Medical University,

Taipei, Taiwan

F. Galluccio

e-mail: felice.galluccio@momarc.org

M. Fajardo Perez

Department of Anesthesia and Chronic Pain, Móstoles University Hospital, Móstoles, Madrid, Spain

e-mail: mfajardoperez@yahoo.es
SHAC, alone (30 patients) or in combination with a suprascapular nerve block (SSnb: 25 patients) or with pectoralis and serratus plane block (PECS-2: 20 patients). All blocks were performed with $0.2 \%$ ropivacaine plus $8 \mathrm{mg}$ dexamethasone. All patients were treated with threeweekly physiotherapy sessions for the following 2 weeks and then with home exercises.

Results: The post-procedural analgesic effect was strong in all groups, with a mean change in numeric rating scale (NRS) values of -6.05 in group $1,-6.25$ in group 2 , and -6.19 in group 3 $(p<.0001)$, allowing all patients to complete an immediate physiotherapy session. Only a few

\section{E. Yamak Altinpulluk}

Department of Outcomes Research Consortium, Anesthesiology Institute Cleveland Clinic,

Cleveland, OH, USA

E. Yamak Altinpulluk

Anaesthesiology Clinical Research Office, Ataturk University, Üniversite Atatürk Üniversitesi

Kampüsü, Yakutiye, Erzurum, Turkey

e-mail: ece.yamak@momarc.org

J.-D. Hou

Division of Anesthesiology, Hualien Armed Forces

General Hospital, Hualien, Taiwan

J.-D. Hou · J.-A. Lin

Department of Anesthesiology, School of Medicine, National Defense Medical Center, Taipei, Taiwan

J.-D. Hou

e-mail: JinDeHou805@gmail.com 
patients needed to repeat the procedure 1 week after the first treatment for the recurrence of pain. From the treatment to the end of the follow-up, we noted a further drop in mean pain NRS values of 1.90 in group 1 and 1.80 in groups 2 and 3. No difference in effect over time was observed among the different groups. No adverse event or motor block was recorded.

Conclusion: This study demonstrates that the SHAC, alone or in combination with other peripheral nerve blocks, is an attractive alternative for shoulder pain management, especially when physiotherapy is required to recover shoulder function.

Keywords: Interfascial plane block; Pericapsular block; Shoulder anterior capsular block

\section{Key Summary Points}

\section{Why carry out this study?}

Shoulder pain is a common disorder with a high prevalence in the general population, and postoperative shoulder pain is among the most intense, resulting invariably in a poor outcome.

The shoulder anterior capsular block is a recently described technique that targets an interfascial and pericapsular space.

The current study evaluated the efficacy of the shoulder anterior capsular block, combined with other peripheral nerve blocks, in relieving shoulder pain while avoiding motor block and allowing for an early rehabilitation program.

\section{J.-A. Lin}

Department of Anesthesiology, School of Medicine, College of Medicine, Taipei Medical University, Taipei, Taiwan

J.-A. Lin

Pain Research Center, Wan Fang Hospital, Taipei

Medical University, Taipei, Taiwan

J.-A. Lin $(\bowtie)$

Department of Anesthesiology, Wan Fang Hospital,

Taipei Medical University, No. 111, Sec. 3,

Xinglong Rd., Wenshan Dist., Taipei City 116,

Taiwan

e-mail: juian.lin@tmu.edu.tw

\section{What was learned from the study?}

This study suggests that the shoulder anterior capsular block, alone or in combination with other blocks, provides a rapid analgesic effect without motor block, allowing for immediate rehabilitation.

\section{INTRODUCTION}

Shoulder pain is a common musculoskeletal disorder, and the lifetime prevalence of shoulder pain in the general population is as high as $67 \%$, significantly affecting patients' ability to work, participation in sport, and activities of daily living such as driving, dressing, brushing hair, and even eating [1]. Open or arthroscopic postoperative shoulder pain is very frequent and often intense [2], even greater than that in hip and knee arthroplasty [3]; thus pain relief after surgery, as well as in chronic pain conditions, continues to be a major challenge for physicians. Poor pain management is invariably reflected in a poor outcome, with longer hospitalization and functional recovery times and an inability to participate in rehabilitation programs, posing a serious problem for health systems and leading to poor quality of life for the patients $[4,5]$. Despite the growing awareness of opioid misuse, opioid monotherapy remains the foundation of postsurgical pain therapy, although opioids are often unable to reduce pain significantly, especially during physiotherapy sessions, and at therapeutic dosages, which have many side effects [6]. In the chronic painful shoulder, it is not uncommon to find a multifactorial etiology of pain, especially in rheumatic or degenerative diseases [7], traumatic injuries, stiff or frozen shoulders, avascular necrosis, nerve entrapment, and complex regional pain syndromes [8-10], where injury can affect multiple structures. The nociception-motor interaction should not be underestimated as a key mechanism in persistent pain and its chronicity, especially in a joint 
with such a wide range and complexity of movement as the shoulder. Therefore, achieving pain relief while avoiding motor block allows us not only to provide an early rehabilitation program in the postoperative period, but also to control the chronic nociceptive activation secondary to pain-induced movement [11].

Ultrasound (US)-guided regional anesthesia has become extremely popular not only for surgical anesthesia but also for pain management, especially in patients with multiple comorbidities, avoiding complications associated with general anesthesia (airway difficulties, hemodynamic instability, etc.) and ensuring excellent pain control, reducing perioperative consumption of opioids and their side effects, and improving patient satisfaction. Among these techniques, the most commonly used is the suprascapular nerve block (SSnb) [8, 12], which accounts for about $70 \%$ of the sensory innervation of the shoulder. Because of the difficulty in effectively reducing pain due to the complexity of shoulder innervation, studies have explored selective axillary nerve blocks with a posterior approach [13] or from the axillae $[14,15]$. These techniques are not always technically easy to perform, and in many cases they cannot cover all axillary nerve branches, especially the glenohumeral articular branch, emerging proximally to the quadrangular space in a high percentage of cases, as described by Gonzalez-Arnay et al. [16], so they are not always effective.

By combining the SSnb with a recently described new block, the shoulder anterior capsular block (SHAC), we can reach all our targets. The SHAC [17-19] is the combination of two different blocks. The first block is the interfascial space between the deep layer of the deltoid fascia and the superficial layer of the subscapularis fascia, anterior to the subscapularis myotendinous junction. Thanks to this interfascial space, we can reach both the axillary nerve and the subscapular nerves, the lateral pectoral nerve, and the musculocutaneous nerve.

The current challenge in the multimodal approach to chronic or postoperative shoulder pain is not only pain relief, but achieving it while maintaining adequate motor function to allow early physiotherapy and rehabilitation and preventing reflex muscle spasticity. Furthermore, it is essential that these techniques are safe and repeatable, and can also be performed in an outpatient setting, facilitating hospital discharge to make them accessible to a greater number of patients.

This works aims to verify whether the SHAC block, combined with the other peripheral nerve blocks, is effective in relieving shoulder pain, avoiding motor block and allowing an early rehabilitation program.

\section{METHODS}

\section{Study Design and Patients}

This was a single-center retrospective chart review of 75 consecutive patients with painful shoulder who underwent a SHAC at the office of the principal author during the previous year in Firenze (Italy).

All patients who had undergone SHAC, alone or in combination with other peripheral nerve blocks, after the failure of standard pain treatments (opioids, steroids, or nonsteroidal anti-inflammatory drugs) and who had not recovered shoulder motion with standard physiotherapy, for whom complete data were available for the observation period, and who were over 18 years of age and provided informed consent were included in this analysis.

Ethics board approval was not required for a labeled procedure and anonymized data of patients already included in a registry dedicated

Table 1 Descriptive analysis categorical variables overall

\begin{tabular}{lll}
\hline Group & Gender & $\boldsymbol{n}(\%)$ \\
\hline SHAC (1) & Female & $16(53.33 \%)$ \\
& Male & $14(46.67 \%)$ \\
SHAC + SSnb (2) & Female & $11(44 \%)$ \\
& Male & $14(56 \%)$ \\
SHAC + PECS-2 (3) & Female & $20(100 \%)$ \\
\hline
\end{tabular}


to locoregional treatment. Written informed consent for data collection and publication was obtained from all participating patients.

Patient data included in this study were subdivided according to the treatment performed: group 1 (30 patients), who were treated only with SHAC; group 2 (25 patients), treated with SHAC + SSnb; and group 3 (20), treated with SHAC + pectoralis and serratus plane block 2 (PECS-2) block (Table 1).

\section{Selection of Treatment According to the Diagnosis}

Treatment was selected based on the patient's diagnosis and associated pathologies. All patients were evaluated by ultrasonography with standard [20], dynamic [21], and elastosonography scans [22]. The combination of SHAC and SSnb was performed in patients with shoulder pain and functional limitation and with rotator cuff injury or high-grade osteoarthritis. Patients with shoulder pain associated with breast surgery (breast cancer with expander placement) [23] were treated with the SHAC + PECS-2 combination. Painful shoulders that did not have significant rotator cuff tears or high-grade glenohumeral osteoarthritis were treated with SHAC block only (Table 2).

All patients were treated with three-weekly physiotherapy sessions for the next 2 weeks and then with home exercises, with the first session 20 min after the end of the procedure.

\section{Description of the Procedures}

For the SHAC block, with the patient in a beachchair position and with the arm in extension, the subscapularis muscle is stretched posteriorly and becomes easily visible in the area below the deltoid fascia. With external rotation and abduction, the coracobrachialis and the biceps brachii muscles are displaced, allowing the visualization of the interfascial space between the deep lamina of the deltoid muscle fascia and the superficial lamina of the subscapularis fascia. The myotendinous nature of the lateral insertion of the subscapularis muscle produces a mixed echogenicity, with a characteristic dotted aspect that facilitates its localization in the US below the deltoid fascia (Fig. 1). Once the

Table 2 Descriptive analysis of first group: continuous variables overall

\begin{tabular}{lrrrrrrrr}
\hline Variable & Mean & SD & Q1 & Median & Q3 & Min & Max & $N$ \\
\hline Age (years) & 60.77 & 5.99 & 56.00 & 62.50 & 65.00 & 50.00 & 70.00 & 30 \\
Pain (months) & 9.27 & 3.17 & 6.00 & 10.00 & 12.00 & 3.00 & 14.00 & 30 \\
NRS T0 & 8.28 & 0.68 & 7.60 & 8.15 & 8.80 & 7.40 & 9.50 & 30 \\
NRS T1 (PP) & 2.23 & 0.90 & 1.50 & 2.30 & 3.10 & 0.80 & 3.60 & 30 \\
NRS T2 (1W) & 2.86 & 2.01 & 1.20 & 2.50 & 2.90 & 0.10 & 6.80 & 30 \\
NRS T3 (PP) & 1.60 & 0.93 & 1.10 & 1.30 & 1.70 & 0.80 & 3.60 & 7 \\
NRS T4 (1W) & 1.29 & 0.70 & 0.60 & 1.10 & 2.00 & 0.50 & 2.20 & 7 \\
NRS T5 & 0.96 & 0.64 & 0.40 & 0.85 & 1.50 & 0.00 & 1.90 & 30 \\
dNRS 0-1 & -6.05 & 1.04 & -6.80 & -5.90 & -5.40 & -8.40 & -4.00 & 30 \\
dNRS 1-2 & 0.63 & 2.20 & -1.00 & 0.10 & 1.80 & -2.60 & 5.30 & 30 \\
dNRS 2-5 & -1.90 & 1.99 & -2.80 & -1.20 & -0.60 & -6.40 & 0.50 & 30 \\
\hline PP
\end{tabular}

$P P$ immediately post-procedure, $1 W 1$ week after procedure 




Fig. 1 Panoramic view of the interfascial space between the deep layer of deltoid fascia and the superficial layer of the subscapularis fascia. $H H$ humeral head, COR coracoid,

injection into the fascial space is achieved, we can proceed towards the glenohumeral pericapsular space by crossing the subscapularis muscle with the needle. By injecting the pericapsular space, we reach the terminal articular branches indistinctly from their origin. Furthermore, through the Weitbrecht foramen, a natural capsular foramen between the upper and middle glenohumeral ligaments, we also reach the intra-articular space (Fig. 2).

All blocks were performed using an aseptic technique with no sedation. The local anesthetic used was a $0.2 \%$ ropivacaine anesthetic solution of $10-15 \mathrm{~mL}$, which has low toxicity, a rapid onset, and a prolonged anesthetic effect, plus dexamethasone $8 \mathrm{mg}$ as adjuvant.

Since the SHAC block is anterior, in cases treated with the SHAC + SSnb combination, the suprascapular nerve was blocked below the
SSc subscapularis, $B B$ biceps brachii, $C B M$ coracobrachialis, $D M$ deltoid muscle, $A X$ axillary nerve

omohyoid muscle [24]. The PECS-II block was performed as described previously [25].

\section{Outcome Measure}

The primary outcome measure was the reduction in pain intensity as quantified by the numeric rating scale (NRS), ranging from 0 (no pain) to 10 (extremely severe pain). Outcome measurements were performed by the same physician (FG) at baseline (T0), after the procedure (T1), at 1 week (T2), and after 3 months (T5). Patients who suffered a relapse of pain 1 week after the first treatment underwent a second procedure identical to the previous one (T2) and were re-evaluated after the procedure (T3) and at 1 week (T4). Adverse events and motor block were assessed at T1 and T3. 




Fig. 2 SHAC block procedure. $H H$ humeral head, $C O R$ coracoid, $S S c$ subscapularis, $B B$ biceps brachii, $C B M$ coracobrachialis, $D M$ deltoid muscle

Table 3 Descriptive analysis of second group: continuous variables overall

\begin{tabular}{lrlrrrrrr}
\hline Variable & Mean & SD & Q1 & Median & Q3 & Min & Max & $N$ \\
\hline Age (years) & 56.04 & 8.72 & 50.00 & 54.00 & 61.00 & 43.00 & 74.00 & 25 \\
Pain (months) & 8.60 & 3.25 & 5.00 & 9.00 & 12.00 & 3.00 & 13.00 & 25 \\
NRS T0 & 8.51 & 0.69 & 8.10 & 8.40 & 8.90 & 7.30 & 9.70 & 25 \\
NRS T1 (PP) & 2.26 & 0.66 & 1.90 & 2.10 & 2.80 & 0.90 & 3.50 & 25 \\
NRS T2 (1W) & 2.35 & 2.10 & 1.30 & 1.70 & 2.50 & 0.00 & 7.30 & 25 \\
NRS T3 (PP) & 2.18 & 0.65 & 1.75 & 2.00 & 2.60 & 1.60 & 3.10 & 4 \\
NRS T4 (1W) & 1.00 & 0.52 & 0.60 & 1.00 & 1.40 & 0.40 & 1.60 & 4 \\
NRS T5 & 0.54 & 0.49 & 0.20 & 0.40 & 0.90 & 0.00 & 1.70 & 25 \\
dNRS 0-1 & -6.25 & 0.82 & -6.90 & -6.10 & -5.70 & -7.80 & -4.70 & 25 \\
dNRS 1-2 & 0.08 & 1.92 & -1.20 & -0.80 & 0.30 & -2.20 & 4.60 & 25 \\
dNRS 2-5 & -1.80 & 2.20 & -2.10 & -1.00 & -0.50 & -6.90 & 1.20 & 25 \\
\hline
\end{tabular}

$P P$ immediately post-procedure, $1 \mathrm{~W} 1$ week after procedure

\section{Statistical Analyses}

Data capture was $100 \%$ up to 3 months of follow-up. Descriptive analyses with continuous variables overall are presented in Table 2 for group 1, Table 3 for group 2, and Table 4 for group 3. Continuous variables are described using the mean (SD), and categorical variables using the absolute and relative frequencies. To evaluate the NRS change over time (T1-T0, T2-T1, T5-T2), the paired $t$ test or signed-rank test, according to the Shapiro-Wilk test for 
normality assumption, was used. To evaluate the association between groups, time, and their interaction, a generalized estimating equation (GEE) linear regression model was used.

\section{RESULTS}

Patients included in group 1 had a mean age of $60.77 \pm 5.99$ years, and shoulder pain was present on average for $9.27 \pm 3.17$ months, with a mean NRS at baseline of $8.28 \pm 0.68$ (Table 3). The most frequent pathology was frozen shoulder (15 patients, 50\%), followed by rheumatoid arthritis (8 patients, $26.7 \%$ ), posttraumatic injury (4 patients, $13.3 \%$ ) and diabetes ( 3 patients, 10\%).

The mean age of group 2 was $56.04 \pm 8.72$ years, with a mean NRS at baseline of $8.51 \pm 0.69$ for $8.60 \pm 3.25$ months (Table 4). Rotator cuff tear (15 patients, 60\%) and glenohumeral osteoarthritis (10 patients, $40 \%$ ) were the two causes of shoulder pain in this group.

Group 3 included 20 women with shoulder pain after breast surgery. Mean age was $41.75 \pm 9.97$ years, with pain for
$3.8 \pm 2.04$ months and mean NRS at baseline of $8.38 \pm 0.72$ (Table 5).

The post-procedural analgesic effect was strong in all three groups, with a mean change in NRS values (dNRS $0-1$ ) of -6.05 in group 1 , -6.25 in group 2 , and -6.19 in group 3 $(p<0.0001)$ (Table 6).

Approximately $20 \mathrm{~min}$ after the procedure, all patients underwent and successfully completed a 45-min physiotherapy session for shoulder mobilization.

Table 5 Treatment selection according to diagnosis

\begin{tabular}{ll}
\hline Group & Diagnosis (no.) \\
\hline SHAC (1) & Frozen shoulder (15) \\
& Rheumatoid arthritis (8) \\
& Post-traumatic (4) \\
& Diabetes (3) \\
SHAC + SSn block & Rotator cuff tear (15) \\
& Glenohumeral osteoarthritis (10) \\
SHAC + PECS-2 & Breast surgery (20) \\
\hline
\end{tabular}

Table 4 Descriptive analysis of third group: continuous variables overall

\begin{tabular}{lrlrrrrrr}
\hline Variable & Mean & SD & Q1 & Median & \multicolumn{1}{c}{ Q3 } & Min & Max & $\boldsymbol{N}$ \\
\hline Age (years) & 41.75 & 9.97 & 34.00 & 39.50 & 51.50 & 28.00 & 59.00 & 20 \\
Pain (months) & 3.80 & 2.04 & 2.00 & 3.50 & 6.00 & 1.00 & 7.00 & 20 \\
NRS T0 & 8.38 & 0.72 & 7.80 & 8.50 & 8.90 & 7.40 & 9.60 & 20 \\
NRS T1 (PP) & 2.18 & 0.86 & 1.40 & 2.10 & 2.95 & 0.90 & 3.60 & 20 \\
NRS T2 (1W) & 2.16 & 2.11 & 0.75 & 1.70 & 2.65 & 0.00 & 6.90 & 20 \\
NRS T3 (PP) & 1.80 & 0.66 & 1.10 & 1.90 & 2.40 & 1.10 & 2.40 & 3 \\
NRS T4 (1W) & 1.80 & 0.30 & 1.50 & 1.80 & 2.10 & 1.50 & 2.10 & 3 \\
NRS T5 & 0.36 & 0.49 & 0.00 & 0.20 & 0.50 & 0.00 & 1.60 & 20 \\
dNRS 0-1 & -6.19 & 1.11 & -7.35 & -6.20 & -5.40 & -7.90 & -3.80 & 20 \\
dNRS 1-2 & -0.02 & 2.26 & -1.80 & -0.45 & 1.20 & -2.80 & 5.40 & 20 \\
dNRS 2-5 & -1.80 & 1.98 & -2.40 & -1.50 & -0.65 & -6.90 & 0.50 & 20 \\
\hline
\end{tabular}

$P P$ immediately post-procedure, $1 W 1$ week after procedure 
Only seven patients in the first group (23.3\%), four in the second group (16\%), and three in the third group (15\%) needed to repeat the block 1 week after the first treatment for recurrence of pain in the days following the first shoulder mobilization.

The procedure was performed in the same way as the previous one, without any changes. From treatment to the end of the follow-up period, we noted a further drop in mean pain NRS values (dNRS 2-5) of 1.90 in group 1 and 1.80 in groups 2 and 3 (Table 6).

The GEE linear regression model data analysis also showed that there were no statistically significant differences among the three subgroups (SHAC, SHAC + SSnb, SHAC + PECS-II). In fact, from the estimation of the coefficients and the analysis of type 1 variance, it is evident that the only difference was over time, while there was no difference in effect over time in the different groups (Table 7).

All patients completed physiotherapy and, at the end of the 3-month observation period, all recovered joint function and range of movement consistent with the underlying pathology.

Finally, no adverse events or motor block were recorded during the whole observation period.

\section{DISCUSSION}

This retrospective data analysis provides preliminary data to support the efficacy of the SHAC, alone or in combination with other peripheral nerve blocks (SSnb, PECS-II), in relieving shoulder pain and avoiding motor block, thus allowing an early rehabilitation program.

Patients undergoing this procedure experienced a noticeable reduction in pain of about 6 points on a 0-10 scale, which allowed for immediate mobilization. To proceed safely and effectively with shoulder mobilization, it is essential to reduce pain, without eliminating it, and to avoid motor block.

In our study, only a small fraction of the patients treated suffered a recurrence of pain at 1 week after the first procedure. These cases were therefore retreated with the same technique, this time obtaining a lasting result.

Finally, we demonstrated that there were no significant differences between the group treated with SHAC alone or in combination with other peripheral blocks (SSnb and PECS-II), and that the only significant difference was over time, confirming the efficacy of these treatments in the control of shoulder pain.

Given the anatomical complexity of the shoulder and its innervation, it is necessary in

Table 6 NRS change over time per group

\begin{tabular}{llllll}
\hline Group & Parameter & Mean change & 95\% LCL & 95\% UCL & $\boldsymbol{p}$ value \\
\hline SHAC (1) & dNRS 0-1 & -6.05 & -6.44 & -5.66 & $<0.0001$ \\
& dNRS 1-2 & 0.63 & -0.19 & 1.45 & 0.1270 \\
SHAC + SSnb (2) & dNRS 2-5 & -1.90 & -2.64 & -1.15 & $<0.0001$ \\
& dNRS 0-1 & -6.25 & -6.59 & -5.91 & $<0.0001$ \\
& dNRS 1-2 & 0.08 & -0.71 & 0.88 & 0.4076 \\
SHAC + PECS-2 (3) & dNRS 2-5 & -1.80 & -2.71 & -0.89 & $<0.0001$ \\
& dNRS 0-1 & -6.19 & -6.71 & -5.67 & $<0.0001$ \\
& dNRS 1-2 & -0.02 & -1.08 & 1.03 & 0.9610 \\
& dNRS 2-5 & -1.80 & -2.73 & -0.87 & $<0.0001$ \\
\hline
\end{tabular}

$L C L$ lower confidence limit, $U C L$ upper confidence limit 
Table 7 Linear regression to evaluate associations between groups, time, and interaction

\begin{tabular}{|c|c|c|c|c|c|c|c|}
\hline \multicolumn{8}{|c|}{ Analysis of the estimates of the maximum likelihood parameters } \\
\hline \multicolumn{2}{|c|}{ Parameter } & \multirow{2}{*}{$\begin{array}{l}\text { Estimate } \\
8.2810\end{array}$} & \multirow{2}{*}{$\begin{array}{l}\text { Standard error } \\
0.2634\end{array}$} & \multicolumn{2}{|c|}{ Wald $95 \%$ confidence limits } & \multirow{2}{*}{$\begin{array}{l}\text { Wald chi-square } \\
988.67\end{array}$} & \multirow{2}{*}{$\begin{array}{l}\text { Pr }>\text { chi-square } \\
<0.0001\end{array}$} \\
\hline Interc & & & & 7.7648 & 8.7971 & & \\
\hline G & 2 & 0.3286 & 0.3725 & -0.4014 & 1.0586 & 0.78 & 0.3777 \\
\hline G & 3 & 0.0940 & 0.3771 & -0.6450 & 0.8331 & 0.06 & 0.8030 \\
\hline $\mathrm{T}$ & 1 & -6.1960 & 0.3771 & -6.9350 & -5.4569 & 269.99 & $<0.0001$ \\
\hline $\mathrm{T}$ & 2 & -5.3560 & 0.3771 & -6.0950 & -4.6169 & 201.75 & $<0.0001$ \\
\hline $\mathrm{T}$ & 5 & -7.2810 & 0.3771 & -8.0200 & -6.5419 & 372.83 & $<0.0001$ \\
\hline $\mathrm{G}^{*} \mathrm{~T}$ & 21 & -0.1136 & 0.5333 & -1.1588 & 0.9316 & 0.05 & 0.8313 \\
\hline $\mathrm{G}^{*} \mathrm{~T}$ & 22 & -0.7086 & 0.5333 & -1.7538 & 0.3366 & 1.77 & 0.1839 \\
\hline $\mathrm{G}^{*} \mathrm{~T}$ & 25 & -0.7436 & 0.5333 & -1.7888 & 0.3016 & 1.94 & 0.1632 \\
\hline $\mathrm{G}^{*} \mathrm{~T}$ & 31 & 0.0060 & 0.5365 & -1.0456 & 1.0575 & 0.00 & 0.9911 \\
\hline $\mathrm{G}^{*} \mathrm{~T}$ & 32 & -0.8590 & 0.5365 & -1.9106 & 0.1925 & 2.56 & 0.1093 \\
\hline $\mathrm{G}^{*} \mathrm{~T}$ & 35 & -0.7340 & 0.5365 & -1.7856 & 0.3175 & 1.87 & 0.1713 \\
\hline Scale & & 1.2069 & 0.0549 & 1.1040 & 1.3193 & & \\
\hline
\end{tabular}

Linear regression statistics for type 1 analysis

\begin{tabular}{lllll}
\hline Origin & $\mathbf{2}^{*}$ Log likelihood & DF & Chi-square & Pr $>$ chi-square \\
\hline Intercept & -1253.0574 & & & \\
G & -1252.4755 & 2 & 0.58 & 0.7475 \\
$\mathrm{~T}$ & -782.9914 & 3 & 469.48 & $<0.0001$ \\
$\mathrm{G}^{*} \mathrm{~T}$ & -777.7770 & 6 & 5.21 & 0.5166 \\
\hline
\end{tabular}

$G$ Group, $T$ Time

some cases to combine nerve blocks to obtain the desired effect. In fact, in these patients, SSnb was performed in rotator cuff injuries or highgrade osteoarthritis, and PECS-II in patients with shoulder pain secondary to breast surgery, where pain is originated mainly by chronic spastic retraction and contraction of the subscapularis and pectoralis minor muscles, with consequent limitation of abduction and extra rotation, and anterior tilt of the scapula.

Based on the clinical observation of patients with chronic shoulder pain or in the postoperative period, we have noticed that in addition to the pain arising from the injured structures, an alteration of muscle function is invariably present, with marked limitation of movements due, in many cases, to the reflex spasticity and protective posture. This condition can be explained by the nociceptor-motor interaction, which causes a progressive alteration of the motor pattern that perpetuates or may even worsen the pain over time.

Nociception affects both efferent and afferent pathways (motor output and proprioception, respectively). Activated neurons in the somatosensory cortex produce a pain-dependent inhibitory input to the primary motor cortex, and tonic muscle nociception also results in long-lasting inhibition of the motor cortex. This event occurs immediately in 
response to pain, but it fades once levels of perceived pain become stable over time, leading to a change in motor behavior, with a different pattern of muscle activation. This includes reduced function, complete avoidance of a movement, or muscle spasticity [11].

Changes in motor control can perpetuate injury and pain if the altered motor strategy or movement leads to excessive loading of tissues. Thus, from a motor-sparing perspective, especially for early mobilization, we must also cover the innervation of all muscles involved in shoulder movement (Fig. 3).

It is also important to remember that in the postoperative period, such as rotator cuff repair, some of the pain that patients experience during early mobilization is probably also due to the presence of scar adhesions, so starting mobilization as soon as possible could guarantee a better outcome. Finally, quickly achieving active mobilization of the painful limb is also associated with greater patient satisfaction, with an improvement in the quality of life $[26,27]$.

Cervical root nerve blocks, such as the interscalene brachial plexus block, is considered the gold standard for providing optimal analgesia and is one of the most commonly used techniques in postoperative analgesia in the surgical setting; however, it is hampered by frequent complications, ranging from accidental epidural anesthesia, to vertebral artery injection, paralysis of the phrenic nerve, pneumothorax, brachial plexus injury, and extended motor block $[28,29]$, and a more specific and peripheral blocking technique would be more suitable.

The SSnb is well known and frequently used in pain clinics as a safe and effective alternative to interscalene block [12], but sometimes does not provide adequate analgesia because the

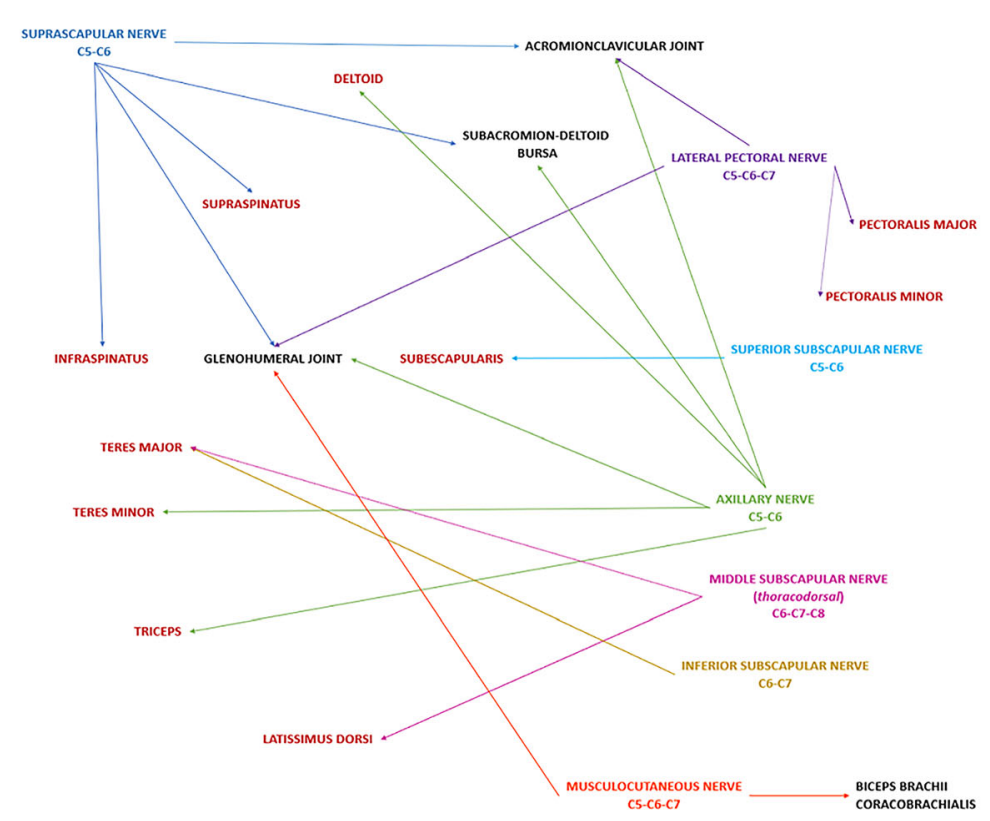

\section{A}

Fig. 3 A The shoulder complex innervation. B Anterior aspect of the shoulder. C Posterior aspect of the shoulder. aSm anterior scalene muscle, $p S m$ posterior scalene muscle, $O M m$ omohyoid muscle, Clav clavicle, $D m$ deltoid muscle, $\mathrm{PMm}$ pectoralis major muscle, $\mathrm{Pmm}$ pectoralis minor muscle, $S S b$ subscapular bursa, $S S m$ subscapularis muscle,

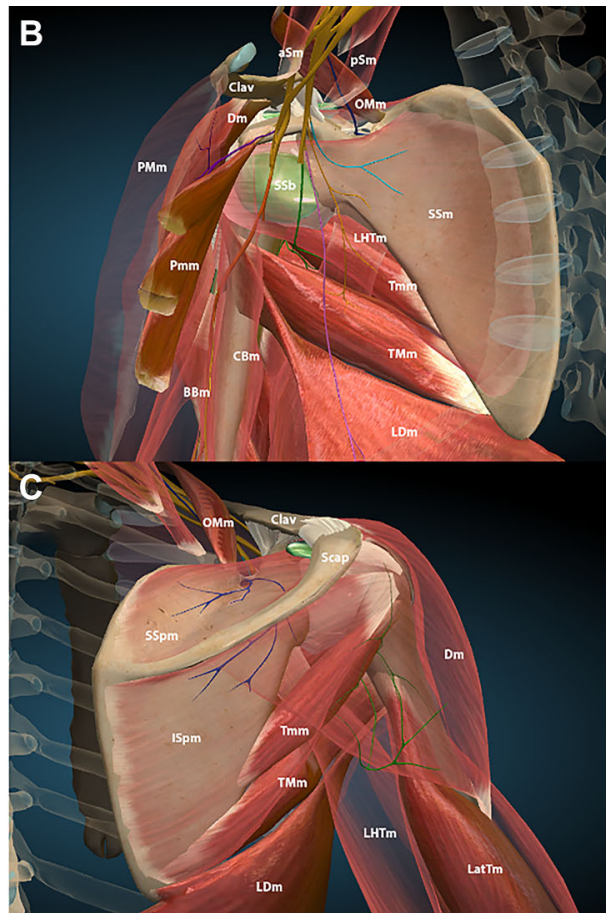

LHTm lateral head of triceps muscle, Tmm teres minor muscle, $T M m$ teres major muscle, $C B m$ coracobrachialis muscle, $B B m$ biceps brachii muscle, $L D m$ latissimus dorsi muscle, Scap scapula, SSpm supraspinatus muscle, ISpm infraspinatus muscle, LatTm long head of triceps, latTm lateral head of triceps 
axillary nerve, the lateral pectoral nerve, and sometimes the musculocutaneous nerve also contribute to the complex innervation of the shoulder joint $[16,30]$. The axillary nerve block through the posterior approach, introduced by Rothe [13], is neither easy to perform nor effective, because the anatomical structures are not always recognizable and because of the articular branches that innervate the glenohumeral joint branch out proximally on the quadrilateral space of Velpeau [16]. More recently, Chang and Feigl described two different ultrasound-guided approaches to track the axillary nerve in the inferior axillary fossa $[14,15]$. In our opinion, this is an interesting approach but should be reserved for skilled professionals because of the complexity of sonoanatomy in recognizing nerve branches and the proximity to the vascular structures that could increase procedural risks.

A recent study published by Ko et al. demonstrated that a combined block of the suprascapular and axillary nerves and the articular branch of the lateral pectoral nerve was not inferior to interscalene block for pain control after arthroscopic repair [31]. Our data agree with the results of that study. Unlike the combined block used in that study, the SHAC includes the superior, middle, and inferior subscapular and the musculocutaneous nerves, completing the innervation of the muscles involved in shoulder disease, especially in chronic conditions.

The SHAC is easy to perform, for two main reasons. The first is that the anatomical structures are easily recognizable even by less experienced sonographers, as the visualization of the coracoid and the myotendinous junction of the subscapularis is among the standard scans of the shoulder and therefore does not require a long learning curve. The second is that a natural way through the articular space is used, the Weitbrecht foramen. Reaching the joint space, in addition to the interfascial and pericapsular spaces, may explain the prolonged analgesic response, especially when a steroid is used as an adjuvant, that is able to modulate and reduce synovial inflammation, deregulate the nerve fibers contained in it, and prolong the analgesic effect.
Another advantage of this technique that should not be underestimated is that the needle is placed in a "safe zone," far enough from any structure that could be involuntarily injured.

This is the first demonstration of the effectiveness of SHAC in shoulder pain management. Although this is a retrospective study, it is a preliminary real-life analysis that informs us about the effectiveness of this treatment, that is, the extent to which this intervention does what it is intended to do in routine circumstances. We believe that this type of study is fundamental because it allows us to identify areas in which investigation is needed and/or to test new hypotheses.

Like all real-life observational studies, this study has limitations, mainly stemming from the lack of randomization, the absence of a control group or a placebo group, and the involvement of only a single center. Given the importance of balancing the variables that could influence the study, enrollment included patients with similar characteristics (shoulder pain) but with different underlying pathologies, divided into three subgroups and treated with SHAC alone or in combination with SSnb or PECS-II. This a priori design was done to improve the consistency of the results, which was later confirmed. More adequately powered and randomized controlled studies are needed to confirm these findings. These studies are ongoing and will be elaborated in the future.

\section{CONCLUSIONS}

In conclusion, this study demonstrates that the SHAC, alone or in combination with other peripheral nerve blocks, is an attractive alternative for shoulder pain management, especially when physiotherapy is required to recover shoulder function. Achieving pain relief, while maintaining motor skills, allows early rehabilitation and the ability to quickly restore normal function of the receptor system involved in the pain mechanism.

Finally, the ease of execution of the SHAC and the low risk of adverse events make it applicable in the outpatient or rehabilitation 
setting as well, allowing a quick hospital discharge and a speedy recovery.

\section{ACKNOWLEDGEMENTS}

We thank Dr. Lorenzo Tofani for his contribution to the statistical analysis, and Guido Baroni and Fabrizio Sigismondi of Fisiotech Lab Studio who performed the physiotherapy sessions. This work was supported by Hualien Armed Forced General Hospital (805-C109-08). We thank all the participants of the study.

Funding. No funding or sponsorship was received for this study or publication of this article.

Authorship. All named authors meet the International Committee of Medical Journal Editors (ICMJE) criteria for authorship for this article, take responsibility for the integrity of the work, and have given their approval for this version to be published.

Authorship Contributions. All authors participated in the interpretation of the data and revising of the manuscript and contributed important intellectual content and approved the final version of the manuscript. Felice Galluccio and Mario Fajardo Perez contributed to the concept and design. The selection and treatment of patients and data collection was performed by Felice Galluccio. Jui-An Lin, Ece Yamak Altinpulluk and Jin-De Hou contributed to the study methodology and data analysis. Felice Galluccio wrote the first draft of the manuscript. All authors commented on previous versions of the manuscript and read and approved the final manuscript.

Disclosures. Felice Galluccio, Mario Fajardo Perez, Ece Yamak Altinpulluk, Jin-De Hou, JuiAn Lin have nothing to disclose.

Compliance with Ethics Guidelines. Ethics board approval was not required for a labeled procedure and anonymized data of patients already included in a registry dedicated to locoregional treatment. Written informed consent for data collection and publication was obtained from all participating patients.

Data Availability. The datasets generated and analyzed during the current study are available from the first author on reasonable request.

Open Access. This article is licensed under a Creative Commons Attribution-NonCommercial 4.0 International License, which permits any non-commercial use, sharing, adaptation, distribution and reproduction in any medium or format, as long as you give appropriate credit to the original author(s) and the source, provide a link to the Creative Commons licence, and indicate if changes were made. The images or other third party material in this article are included in the article's Creative Commons licence, unless indicated otherwise in a credit line to the material. If material is not included in the article's Creative Commons licence and your intended use is not permitted by statutory regulation or exceeds the permitted use, you will need to obtain permission directly from the copyright holder. To view a copy of this licence, visit http://creativecommons.org/licenses/by$\mathrm{nc} / 4.0 /$.

\section{REFERENCES}

1. Luime JJ, Koes BW, Hendriksen IJ, Burdorf A, Verhagen AP, Miedema HS, Verhaar JA. Prevalence and incidence of shoulder pain in the general population; a systematic review. Scand J Rheumatol. 2004;33:73-81.

2. Wilson AT, Nicholson E, Burton L, Wild C. Analgesia for day-case shoulder surgery. Br J Anaesth. 2004;92:414-5.

3. Lindberg MF, Grov EK, Gay CL, et al. Pain characteristics and self-rated health after elective orthopaedic surgery-a cross sectional survey. J Clin Nurs. 2013;22:1242-53. https://doi.org/10.1111/ jocn.121493.

4. McQuay H, Derry S, Wiffen P, Moore A. postoperative pain management: number-needed-to-treat approach versus procedure-specific pain management approach. Pain. 2013;154:180. 
5. Institute of Medicine. Relieving pain in America. National Academies Press; 2011. (ISBN-13.9780-0309-21484-1).

6. Coyne KS, Barsdorf AI, Currie BM, Poon JL, Mazière JY, Pierson RF, Butler SF, Schnoll SH, Farrar JT, Fisher HJ, Franks MJ Jr. Insight into chronic pain in the United States: descriptive results from the Prescription Opioid Misuse and Abuse Questionnaire (POMAQ) validation study. Curr Med Res Opin. 2020;17:1. https://doi.org/10.1080/03007995.2020. 1865889 .

7. Buchbinder R, Staples MP, Shanahan EM, et al. General practitioner management of shoulder pain in comparison with rheumatologist expectation of care and best evidence: an Australian National Survey. PLoS ONE. 2013;8:e61243.

8. Dean BJ, Gwilym SE, Carr AJ. Why does my shoulder hurt? A review of the neuroanatomical and biochemical basis of shoulder pain. Br J Sports Med. 2013;47:1095-104.

9. Kim JS, Park J-Y, Kwon KB, Chung SW. Shoulder stiffness in patients with rotator cuff tears. Arthrosc Orthop Sports Med. 2016;3:32-9.

10. Zhang K, de Sa D, Kanakamedala A, Sheean AJ, Vyas D. Management of concomitant preoperative rotator cuff pathology and adhesive capsulitis: a systematic review of indications, treatment approaches, and outcomes. Arthroscopy. 2019;35(3):979-93.

11. Struyf F, Lluch E, Falla D, et al. Influence of shoulder pain on muscle function: implications for the assessment and therapy of shoulder disorders. Eur J Appl Physiol. 2015;115:225.

12. Sun C, Ji X, Zhang X, Ma Q, Yu P, Cai X, Yang H. Suprascapular nerve block is a clinically attractive alternative to interscalene nerve block during arthroscopic shoulder surgery: a meta-analysis of randomized controlled trials. J Orthop Surg Res. 2021;16(1):376. https://doi.org/10.1186/s13018021-02515-1.PMID:34116689;PMCID: PMC8194158.

13. Rothe C, Lund J, Jenstrup MT, Lundstrøm LH, Lange KH. Ultrasound-guided block of the axillary nerve: a case series of potential clinical applications. Acta Anaesthesiol Scand. 2012;56(7):926-30.

14. Chang KV, Lin CP, Lin CS, Wu WT, Özçakar L. A novel approach for ultrasound guided axillary nerve block: the inferior axilla technique. Med Ultrason. 2017;19(4):457-61.

15. Feigl G, Aichner E, Mattersberger C, Zahn PK, Avila Gonzalez C, Litz R. Ultrasound-guided anterior approach to the axillary and intercostobrachial nerves in the axillary fossa: an anatomical investigation. Br J Anaesth. 2018;121(4):883-9. https:// doi.org/10.1016/j.bja.2018.06.006.

16. González-Arnay E, Jiménez-Sánchez L, GarcíaSimón D, et al. Ultrasonography-guided anterior approach for axillary nerve blockade: an anatomical study. Clin Anat. 2020;33(4):488-99. https://doi. org/10.1002/ca.23394.

17. Galluccio F, Arnay EG, Salazar C, et al. Re: "Ultrasound-guided block of the axillary nerve: a prospective, randomized, single-blind study comparing interfascial and perivascular injections." Pain Physician. 2020;23(1):E62-4.

18. Yamak Altinpulluk E, Galluccio F, Salazar C, et al. A novel technique to Axillary Circumflex Nerve Block: Fajardo approach [published online ahead of print, 2020 Apr 16]. J Clin Anesth. 2020;64:109826. https://doi.org/10.1016/j.jclinane.2020.109826.

19. Yamak Altinpulluk E, Teles AS, Galluccio F, et al. Pericapsular nerve group block for postoperative shoulder pain: a cadaveric radiological evaluation. J Clin Anesth. 2020;67: 110058. https://doi.org/10. 1016/j.jclinane.2020.110058.

20. Özçakar L, Kara M, Chang KV, Tekin L, Hung CY, Ulaülı AM, Wu CH, Tok F, Hsiao MY, Akkaya N, Wang TG, Çarli AB, Chen WS, De Muynck M. EURO-MUSCULUS/USPRM basic scanning protocols for shoulder. Eur J Phys Rehabil Med. 2015;51(4):491-6 (Epub 2015 Jul 9).

21. Chang KV, Wu WT, Hsu PC, Lew HL, Özçakar L. Clinical tests of the shoulder: accuracy and extension using dynamic ultrasound. Am J Phys Med Rehabil. 2020;99(2):161-9. https://doi.org/10.1097/ PHM.0000000000001311.

22. Chiu YH, Chang KV, Chen IJ, Wu WT, Özçakar L. Utility of sonoelastography for the evaluation of rotator cuff tendon and pertinent disorders: a systematic review and meta-analysis. Eur Radiol. 2020;30(12):6663-72. https://doi.org/10.1007/ s00330-020-07059-2 (Epub 2020 Jul 14).

23. Kim JH, Kim SH, Kim HR, Lee SH, Yoon SY, Yang JH, Yoo YB, Park KS, Nam SE, Hong S, Min HK. Ultrasonographic evaluation of chronic shoulder pain after breast cancer surgery: single center, crosssectional study. Sci Rep. 2020;10(1):16792. https:// doi.org/10.1038/s41598-020-73769-8.

24. Sehmbi H, Johnson M, Dhir S. Ultrasound-guided subomohyoid suprascapular nerve block and phrenic nerve involvement: a cadaveric dye study. Reg Anesth Pain Med. 2019;44(5):561-4. https://doi. org/10.1136/rapm-2018-100075. 
25. Blanco R, Fajardo M, Parras Maldonado T. Ultrasound description of Pecs II (modified Pecs I): a novel approach to breast surgery. Rev Esp Anestesiol Reanim. 2012;59:470-5.

26. Shen C, Tang ZH, Hu JZ, Zou GY, Xiao RC, Yan DX. Does immobilization after arthroscopic rotator cuff repair increase tendon healing? A systematic review and meta-analysis. Arch Orthop Trauma Surg. 2014;134(9):1279-85. https://doi.org/10.1007/ s00402-014-2028-2.

27. Kjær BH, Magnusson SP, Warming S, Henriksen M, Krogsgaard MR, Juul-Kristensen B. Progressive early passive and active exercise therapy after surgical rotator cuff repair-study protocol for a randomized controlled trial (the CUT-N-MOVE trial). Trials. 2018;19(1):470. https://doi.org/10.1186/s13063018-2839-5.

28. Hussain N, Goldar G, Ragina N, Banfield L, Laffey JG, Abdallah FW. Suprascapular and interscalene nerve block for shoulder surgery: a systematic review and meta-analysis. Anesthesiology. 2017;127(6):998-1013.

29. Abdallah FW, Halpern SH, Aoyama K, Brull R. Will the real benefits of single-shot interscalene block please stand up? A systematic review and metaanalysis. Anesth Analg. 2015;120:1114-29.

30. Akita K, Kawashima T, Shimokawa T, Sato K, Sato T. Cutaneous nerve to the 487 subacromial region originating from the lateral pectoral nerve. Ann Anat. 2002;488(184):15-9.

31. Ko SH, Park SH, Jang SM, Lee KJ, Kim KH, Jeon YD. Multimodal nerve injection provides noninferior analgesic efficacy compared with interscalene nerve block after arthroscopic rotator cuff repair. J Orthop Surg (Hong Kong). 2021;29(2): $23094990211027976 . \quad$ https://doi.org/10.1177/ 23094990211027974 